\title{
A suitable segmentation methodology based on pixel similarities for landmine detection in IR images
}

\author{
Dr. G.Padmavathi ${ }^{1}$, \\ Department of Computer Science, \\ Avinashilingam University for \\ Women, Coimbatore, India \\ ganapathi.padmavathiegmail.
}

\author{
Dr. P. Subashini ${ }^{2}$, \\ Department of Computer Science, \\ Avinashilingam University for \\ Women, Coimbatore, India \\ mail.p.subashinilgmail.com
}

\author{
Ms. M. Krishnaveni ${ }^{3}$ \\ Department of Computer Science, \\ Avinashilingam University for \\ Women, Coimbatore, India \\ krishnaveni.rdegmail.com
}

\begin{abstract}
Identification of masked objects especially in detection of landmines is always a difficult problem due to environmental inference. Here, segmentation phase is highly concentrated by performing an initial spatial segmentation to achieve a minimal number of segmented regions while preserving the homogeneity criteria of each region. This paper aims in evaluating similarities based segmentation methods to compose the partition of objects in Infra-Red images. The output is a set of non-overlapping homogenous regions that compose the pixels of the image. These extracted regions are used as the initial data structure in feature extraction process. Experimental results conclude that h-maxima transformation provides better results for landmine detection by taking the advantage of the threshold. The relative performance of different conventional methods and proposed method are evaluated and compared using the Global Consistency Error and Structural Content. It proves that h-maxima gives significant results that definitely facilitate the landmine classification system more effectively.
\end{abstract}

Keywords- Segmentation, Global Consistency error, h-maxima, threshold, Landmine detection

\section{INTRODUCTION}

The signature of buried land mine in IR images varies significantly depending on external parameters such as weather, soil moisture, solar radiation, burial depth, and time[7]. By literature [Oscar González Merino] [11] the working of many image-based landmine detection algorithms, it is concluded that the fundamental challenges arise from the fact that the mean spectral signatures of disturbed soil areas that indicate mine presence are nearly always very similar to the signatures of mixed background pixels that naturally occur in heterogeneous scenes composed of various types of soil and vegetation [1][12]. Mine detection using infrared techniques is primarily based on exploiting temperature differences between pixels on the mines and background pixels [2]. Thus, there is always a need for robust algorithm that has the capability to analyze the pattern of distribution of the pixels to separate pixels of the mines from background pixels. Here the segmentation methods are evaluated with performance metrics [3]. Experimental results show that h-maxima transformation is more adoptable for IR target images. The paper is organized as follows: Section 2 deals with the need for pixel based segmentation for IR images. Section 3 converses the comparison of segmentation methods and the subjective assessment of the approach Section 4 explores the performance evaluation of the methods. The paper ends with observations on future work and some conclusions.

\section{PRELIMINARIES OF SEGMENTATION}

There are many unsupervised and supervised segmentation algorithms [6]. They only use low-level features, e.g. intensity and texture, to generate homogeneous patches from an input image. Four categories for segmentation are: histogram shape based methods, where, for example, the peaks, valleys and curvatures of the smoothed histogram[4]. Clustering based methods, where the gray level samples are clustered in two parts as background and foreground (object), or alternately are modeled as a mixture of two Gaussians. Entropy based methods results in algorithms that use the entropy of the foreground and background regions, the cross entropy between the original and binarized image, etc. Object attribute-based methods search a measure of similarity between the gray level and the binarized images, such as fuzzy shape similarity, edge coincide, etc. This paper quantifies the segmentation methods based on the similarities of the pixels including the intensity and the object structure.

\section{Similarities Based SeGmentation TECHNiQUES}

Segmentation is a pre-process which partitioned image into unique multiple regions, where region is set of pixels. Mathematically segmentation can be defined as follows:

If $\mathrm{I}$ is set of all image pixels, then by applying segmentation we get different unique regions like $\{\mathrm{S} 1, \mathrm{~S} 2, \mathrm{~S} 3, \ldots, \mathrm{Sn}\}$ which when combined formed ' $\mathrm{I}$ ' . Basic formulation is as follows:
(a) $\bigcup_{i=1, n}^{n} S_{i}=I \quad$ where $S_{i} \bigcap S_{j}=\varnothing$
(b) $\mathrm{Si}$ is a connected region, $\mathrm{i}=1,2 \ldots \mathrm{n}$.
(c) $\mathrm{P}(\mathrm{S} \mathrm{i})=$ TRUE for $\mathrm{i}=1,2 \ldots \mathrm{n}$.
(d) $P\left(S_{i} \bigcup S_{j}\right)=F A L S E$ for $i \neq j$. 
Where $\mathrm{P}(\mathrm{Si})$ is a logical predicate defined over the points in set Si.

Condition (a) indicates that segmentation must be complete, every pixel in the image must be covered by segmented regions. Segmented regions must be disjoint. Condition (b) requires that points in a region be connected in some predefined sense like 4- or 8- connected. Condition (c) deals, the properties must be satisfied by the pixels in a segmented region- e.g. $\mathrm{P}\left(\mathrm{S}_{\mathrm{i}}\right)=$ TRUE. if all pixels in $\mathrm{S}_{\mathrm{i}}$ have the same gray level. Last condition (d) indicates that adjacent regions $S_{\mathrm{i}}$ and $\mathrm{S}_{\mathrm{j}}$ are different in the sense of predicate $\mathrm{P}$.

Ever in image processing research there is no common solution to the segmentation problem [6]. One of the main reasons of segmentation algorithms is to precisely segment the image without under or over segmentation. Almost all image segmentation techniques proposed so far are ad hoc in nature. These below are the following approaches of image segmentation taken in this paper and demonstrated with IR images. Given below in fig 1 is the approach taken for image segmentation for IR images.

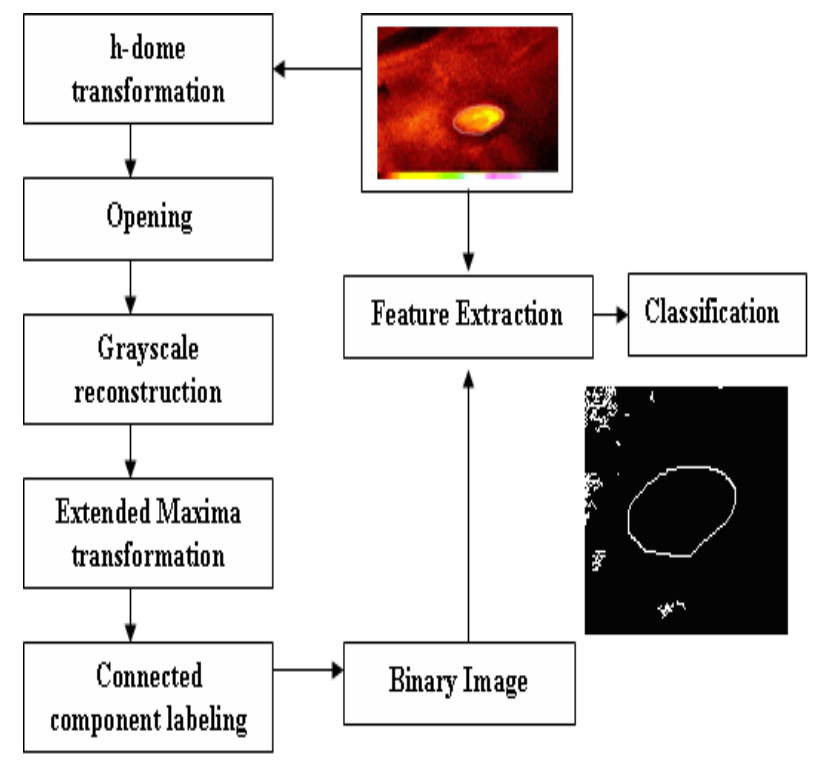

Figure 1: Schematic representation of proposed algorithm for identification of landmine

\section{A. H-maxima algorithm}

Every IR image taken has been implemented with morphological reconstruction, extended maxima transformation using thresholding[10]. The extended maxima transformation is the regional maxima computation of the corresponding $h$ maxima transformation. As a result, it produces a binary image. A connected-component labeling operation is performed, in order to evaluate the characteristics and the location of every object. As a second object reduction step, objects not located within a region of another object, are also discarded, since mine objects are not typically clustered. The region of interest (ie) the target is got by connected component segmentation in which the relevant pixels of the object will be grouped and extorted [8]. The extended-maxima transform computes the regional maxima of the $\mathrm{H}$-maxima transform. Here $\mathrm{H}$ refers to nonnegative scalar. Regional maxima are connected components of pixels with a constant intensity value, and whose external boundary pixels will have a lower value.

\section{B. Kmeans algorithm}

Here $\mathrm{K}$ means is used as a two phase iterative algorithm[9] to minimize the sum of point-to centroid distances, summed over all $\mathrm{k}$ clusters. The first phase uses each iteration that consists of reassigning points to their nearest cluster centroid. The second phase uses points that are individually reassigned. Arbitrarily choose $\mathrm{k}$ data points to act as cluster centers. Until the cluster centers are unchanged following are the steps carried out : Allocate each data -point to cluster whose center is nearest. Replace the cluster centers with the mean of the elements in their clusters end. Clustering in attribute space can lead to unconnected regions in image space (but this may be useful for handling occlusions). The $\mathrm{K}$ means image representation groups all feature vectors from all images into $\mathrm{K}$ clusters and provides a cluster id for every region of image that represents the salient properties of the region. $\mathrm{K}$ means is fast iterative and leads to a local minimum. It looks for unusual reduction in variance. This iterative algorithm has two steps

Assignment step: Assign each observation to the cluster with the closest mean

$$
S_{i}^{(t)}=\left\{X_{j}:\left\|X_{j}-m_{i}^{(t)}\right\|\|\leq\| x_{j}-m^{(t)} \|\right.
$$

Update step: Calculate the new means to be centroid of the observations in the cluster

$$
m_{i}^{(t+1)}=\frac{1}{\left|S_{i}^{(t)}\right|} \sum_{x_{j} \in S(t)_{i}} \mathbf{X}_{j}^{-}
$$

\section{Threshold intensity distribution algorithm}

It can detect object boundaries with low gradient or reduce noise effect in gradient. However, an accurate and stable estimation of intensity distribution is difficult to get from a finite set of 3D image data. To reduce the "shrink" or "expand" effect on segmentation results, gradient information is used to calibrate the estimation of intensity distribution in the following. Overlap of image gradient is computed with the boundaries determined by intensity distribution through introducing a probability offset to intensity distribution. The maximum overlap indicates the optimal boundaries of the interested objects. To restate the problem without losing generality, here it use a mixed Gaussian distribution model.

$$
P(u)=\sum_{k=1}^{n} \pi_{k} P\left(u \mid \lambda_{k} ; \mu_{k}, \sigma_{k}\right) \text {, }
$$


Where $\pi_{k}$ is the prior probability of class $\lambda_{k}$ with $\sum_{k=1}^{n} \pi_{k}=1$, and $\mu_{k}, \sigma_{k}$ are the mean and variance of the Gaussian distribution of the intensity. Intensity distribution inside the region $\Omega$ is in Eq below

$$
P_{i n}(u)=\sum_{k \mid \lambda_{k} \in \Omega} \pi_{k} P\left(u \mid \lambda_{k} ; \mu_{k}, \sigma_{k}\right)
$$

And the intensity distribution of the outside region $\Omega, P_{\text {out }}$, can be obtained in a similar way. Normally for pixel $\mathrm{x}$ on region boundaries with $\mathrm{u}=\mathrm{I}(\mathrm{x})$,

$$
P_{\text {out }}(I(x))-P_{\text {in }}(I(x))=0
$$

Threshold Intensity Distribution is a significant aspect in assigning an individual region around an image[4]. Uneven distribution may lead to assignment of two or more regions to an individual pixel. This method reduces processing time by performing gray-level based segmentation that extracts regions of uniform intensity. Subsequently, it is also possible to estimate motion for the regions. It also reduces the computational load, and the region-based estimator gives robustness to noise and changes of illumination. The segmentation of the reference image is designed to group pixels of similar gray-levels. In intensity distribution, $B(i, j)$ is a binary image (pixel are either 0 or 1 ) created by thresholding $\mathrm{F}(\mathrm{i}, \mathrm{j})$

$$
\begin{aligned}
& B(i, j)=1 \text { if } F(i, j)<t \\
& B(i, j)=0 \text { if } F(I, j)>=t
\end{aligned}
$$

It is assumed that the 1's are the object pixels and the 0's are the background pixels.

The Histogram (h) - gray level frequency distribution of the gray level image $\mathrm{F}$.

$\mathrm{h}_{\mathrm{F}}(\mathrm{g})=$ number of pixels in $\mathrm{F}$ whose gray level is $\mathrm{g}$

$\mathrm{H}_{\mathrm{F}}(\mathrm{g})=$ number of pixels in $\mathrm{F}$ whose gray level is $<=\mathrm{g}$

This method is a probabilistic method that makes parametric assumptions about object and background intensity distributions and then derives "optimal" thresholds.

\section{Boundary based algorithm}

Here the strategy is to consider each control point in turn and move it to the pixel; in its local neighbourhood which gives us the minimum. For a closed boundary it could make the initial estimate surround the object of interest, and add in another term to the objective function to penalize the total length. A difficulty with this type of strategy is the control points. This method traces the exterior boundaries of objects, as well as boundaries of holes inside these objects, in the binary image. A binary image is considered in which the nonzero pixels belong to an object and 0 pixels constitute the background.

Rules that are worked out to guide the process of true corner point localization.

(i) Select those boundary points which bear significantly large cornerity index by eliminating the boundary points which lie on straight line segments bearing negligibly small cornerity index value.

(ii) Since all points on a smooth curve segment are in general associated with almost same cornerity index, and actual corner points bear cornerity index larger than that of their neighbors, it is suggested to select the set of connected points such that the variations in their cornerity indices are considerably large. This rule helps in selecting only the set of points with in the vicinity of actual corner points by eliminating the points on smooth curve segments.

(iii) Select the points which bear local maximum cornerity index as true corner points. It could be noticed that these rules do not require any priori knowledge in locating true corner points.

Thus, the expected point corresponding to a corner point will have a larger shift when compared to other points on the boundary curve. Therefore, the cornerity index of pi is defined to be the Euclidean distance $d$ between the points pi and its expected point pie and is given by

$$
d=\sqrt{\left(x_{i}-x_{i e}\right)^{2}+\left(y_{i}-y_{i e}\right)^{2}}
$$

The cornerity index indicates the prominence of a corner point. The larger the value of the cornerity index of a boundary point, the stronger is the evidence that the boundary point is a corner. 


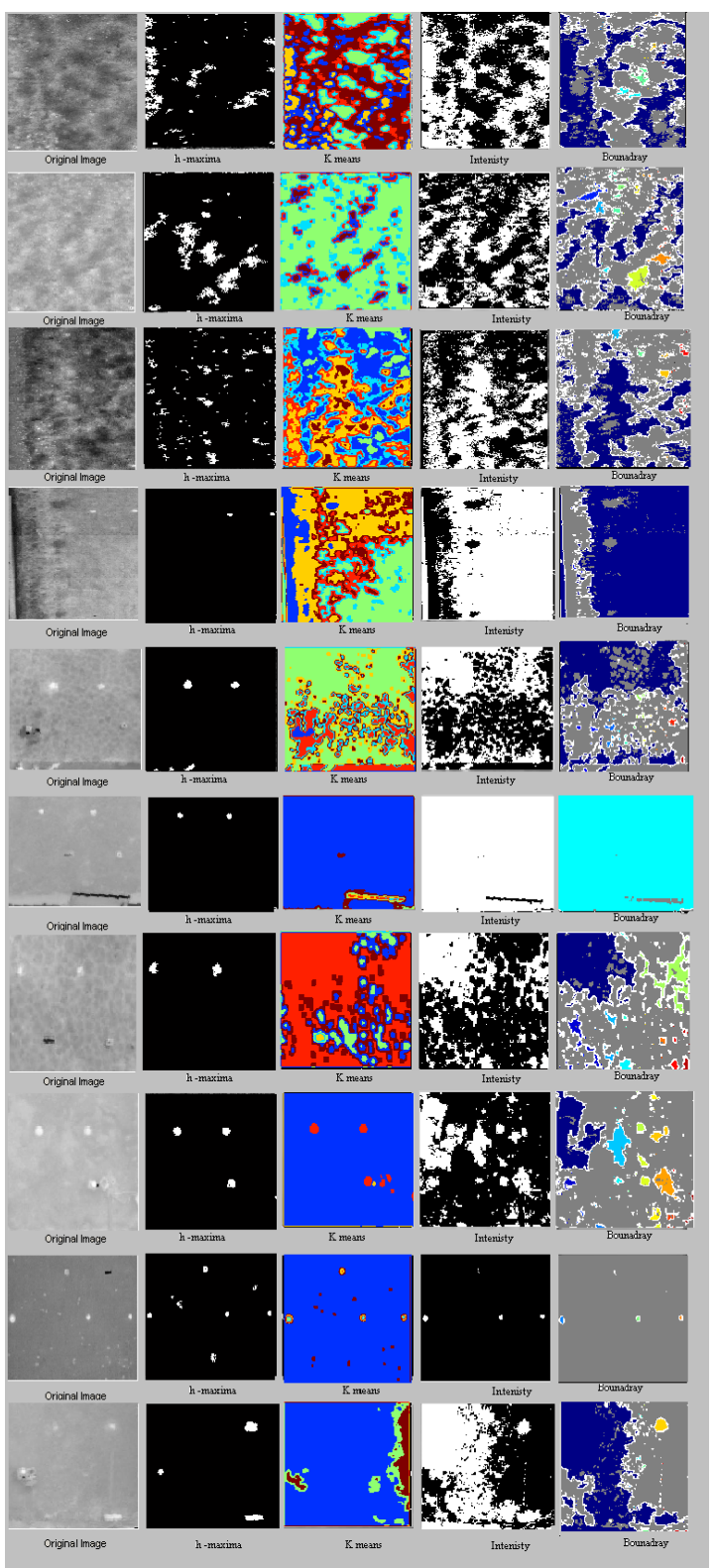

Figure 2: Visual assessment of the segmentation methods

\section{PERFORMANCE EVAlUATION}

The qualitative and quantitative assessment of segmentation results is carried out here for choosing the appropriate approach for a given segmentation task. Similar to the segmentation theory itself, there is no established standard procedure for the evaluation of its results. For this reason evaluation is done using empirical discrepancy method using the relative ultimate measurement accuracy. Global Consistency Error and Structural Content used as the evaluation parameters to control the segmentation process and dynamically a good number of regions are chosen based on local minima in the segmentation evaluation measure[5].The uniqueness of each parameter takes the advantage and disadvantages of IR images.
Global Consistency Error (GCE) forces all local refinements to be in the same direction and is defined as: $\operatorname{GCE}\left(S, S^{\prime}\right)=\frac{1}{N} \min \left\{\sum \operatorname{LRE}\left(S, S^{\prime}, x_{i}\right), \sum \operatorname{LRE}\left(S^{\prime}, S, x_{i}\right)\right\} \cdot(3)$

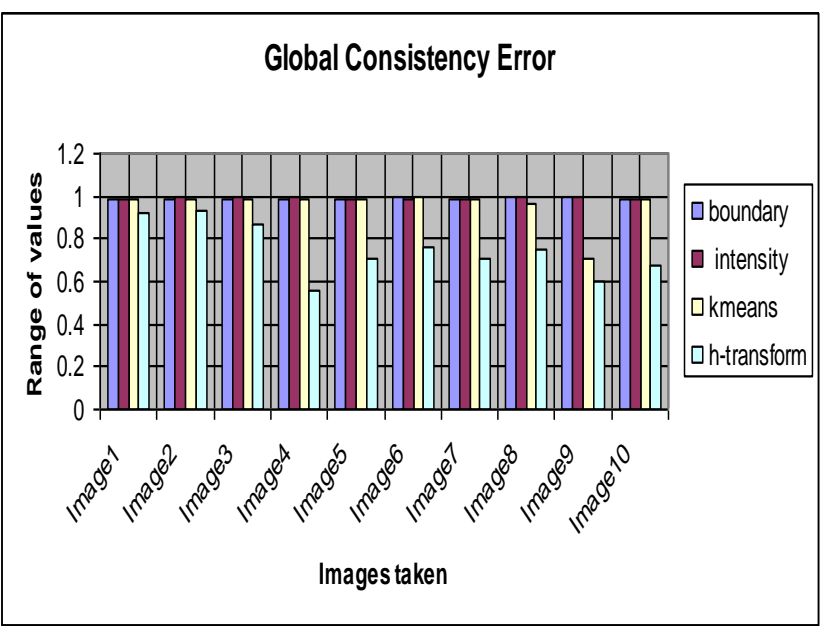

Figure 3: Comparison based on Global Consistency Error

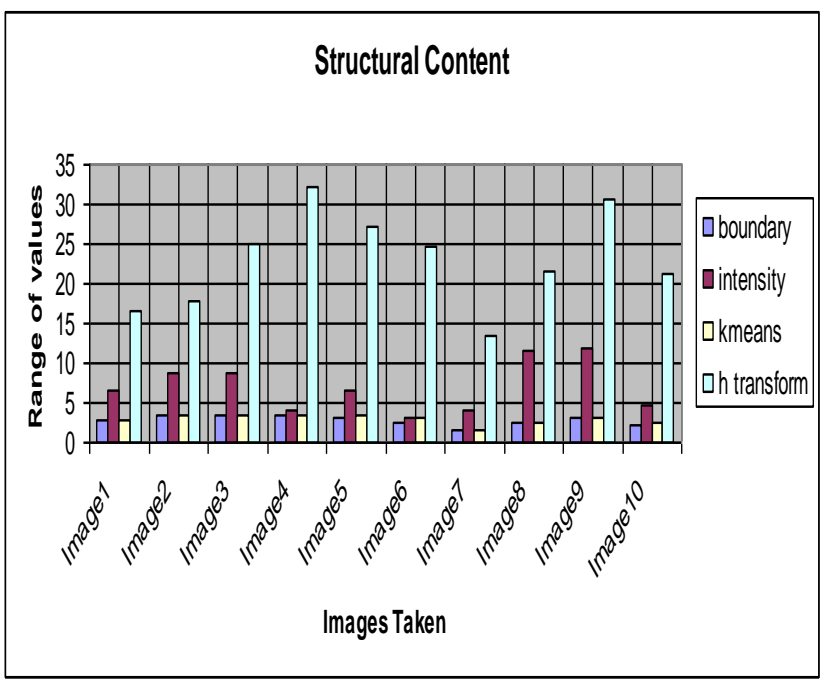

Figure 4: Comparison based on structural content

A structural content is used to find mask structural defects and noise, but are prone to periodic artifacts. This examines to classify image regions hierarchically based on the level of structural content.

$S C=\sum_{j=1}^{M} \sum_{k=1}^{N} x_{j, k^{2}} 2 / \sum_{j=1}^{M} \sum_{k=1}^{N} x_{j, k^{2}}^{\prime} 2 /$

Structural content of a segmented image is calculated by summation of the original image by the division of the segmented image. 
From the above figures 3, 4 the objective evaluation also states that $\mathrm{h}$ maxima transform is the desirable method for landmine detection. The Global consistency error rate should always be low and it is the same for the proposed method in comparatively to the conventional methods. The structural content is high for h-maxima which state that the originality of the object and its information remains the same after the segmentation process.

\section{CONCLUSION}

The method described in this paper provides a relatively simple, extremely fast, and robust method for displaying and performing automatic target identification phase. The result of image segmentation is a set of segments that collectively cover the entire image, or a set of contours extracted from the image. Each of the pixels in a region is similar with respect to some characteristic or computed property, such as color, intensity, or texture. Adjacent regions are significantly different with respect to the same characteristic(s). From this paper it is concluded that h-maxima is very useful in IR image evaluation but that it should probably restrict studies to similar images and similar processing. Simulations are carried out which demonstrates the proposed method is able to successfully localize landmine objects from different sets of real IR images. Nevertheless, this scheme has some limitations because it is not automatic as different parameters have to be adjusted manually. Future work should incorporate the use of high-level image analysis methods for the identification of the true mine objects among the set of the detected mine cues.

\section{REFERENCES}

[1] Frank cremer't, wim de jong and klamer schutte ," processing of polarimetric infrared images for landmine detection",2nd international workshop on advanced gpr. 14-16 may, 2003, the Netherlands

[2] E Ctrmn, W. de long, and K Schutte, "Fusion of polarimetric infrared features and GPR features far landmine detection," in 2nd International Workshop on Advanced Ground Penetrating Radar (IWAGPR), Delft, The Netherlands, May 2003.

[3] Nathir A. Rawashdeh, Shaun T. Love " Hierarchical Image Segmentation by Structural Content" journal of software, vol. 3, no. 2, february 2008

[4] Yongsheng Hu, Qian Chen,"An Infrared Image Preprocessing Method Using Improved Adaptive Neighborhoods ",Proceedings of the 6th World Congress on Intelligent Control and Automation, June 21 - 23, 2006, Dalian, China

[5] Zhou Wang, Alan Conard Bovik, Hamid Rahim Sheik and Erno P Simoncelli, "Image Quality Assessment: From Error Visibility to Structural Similarity", IEEE Trans. Image Processing, and Vol. 13, (2004).

[6] R.M. Haralick, and L.G. Shapiro, "Survey: Image Segmentation Techniques," Computer Vision, Graphics, and Image Processing, Vol. 29, pp. 100-132, 1985.
[7] F. Cremer and W. de Jong and K. Schutte. 'Infrared polarization measurements of surface and buried anti-personnel landmines'. In Preprint Proc. SPIE Vol. 4394, Detection and Remediation Techniques for Mines and Minelike Targets VI, Orlando FL, USA, Apr. 2001.

[8] Yan Sun, Chengyi Sun and Wanzhen Wang, "Color Images Segmentation Using New Definition of Connected Components", Proceedings of ICSP2000.

[9] K.S. Ravichandran,B. Ananthi, "Color Skin Segmentation Using KMeans Cluster" International Journal of Computational and Applied Mathematics ISSN 1819-4966 Volume 4 Number 2 (2009), pp. 153-157

[10] Punam K. Saha, "Optimum Image Thresholding via Class Uncertainty and Region Homogeneity",IEEE transactions on pattern analysis and machine intelligence, vol. 23, no. 7, july 2001

[11] Oscar González Merino "Image analysis of Infrared Polarization measurements of Landmines", Academic year: 2000/01

[12] H. Brunzell, "Clutter Reduction and Object Detection in Surface Penetrating Radar," in Proc. Radar.97 Conf., 1997 pp. 688-691.

\section{AUTHORS PROFILE}

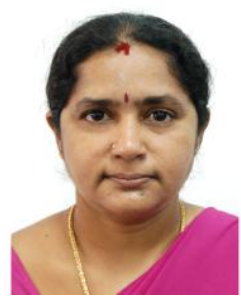

Dr. Padmavathi Ganapathi is the Professor and Head of Department of Computer Science, Avinashilingam University for Women, Coimbatore. She has 23 years of teaching experience and one year Industrial experience. Her areas of interest include Network security and Cryptography and real time communication. She has more than 108 publications at national and International level. She is a life member of many professional organizations like CSI, ISTE, AACE, WSEAS, ISCA, and UWA.

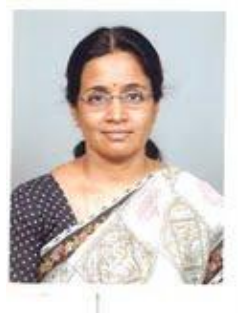

Dr. Subashini is the Associate professor in Department of Computer Science, Avinashilingam Deemed University for Women, Coimbatore. She has 16 years of teaching experience. Her areas of interest include Object oriented technology, Data mining, Image processing, Pattern recognition. She has 55 publications at national and International level.

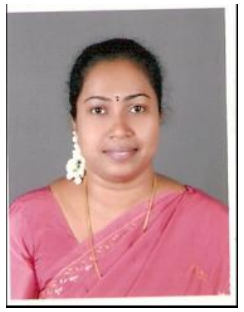

Ms.M.Krishnaveni has 4 Years of Research Experience Working as Research staff in DRDO and pursuing her $\mathrm{Ph} . \mathrm{D}$ in Avinashilingam University for Women, Coimbatore. Her research interest are Image Processing, Pattern Recognition and Neural Networks. She has 24 publications at national and international level. 